\begin{tabular}{|c|c|}
\hline Title & Formation of GaN porous structures with improved structural controllability by photoassisted electrochemical etching \\
\hline Author(s) & Kumazaki, Y usuke; Y atabe, Zenji; Sato, T aketomo \\
\hline Citation & $\begin{array}{l}\text { Japanese Journal of A pplied Physics (JJA P), 55(4), 04EJ12 } \\
\text { https://doi.org/10.7567/JJA P.55.04EJ12 }\end{array}$ \\
\hline Issue Date & 2016-04 \\
\hline Doc URL & http:/hdl .handle.net/2115/64688 \\
\hline Rights & (C) 2016 The Japan Society of A pplied Physics \\
\hline Type & article (author version) \\
\hline File Information & JJA P55_04EJ12.pdf \\
\hline
\end{tabular}

Instructions for use 


\title{
Formation of GaN porous structures with improved structural controllability by photoassisted electrochemical etching
}

\author{
Yusuke Kumazaki, Zenji Yatabe, and Taketomo Sato \\ Research Center for Integrated Quantum Electronics, and Graduate School of Information Science and \\ Technology, Hokkaido University, Sapporo 060-8628, Japan
}

\begin{abstract}
We aimed to develop a photoassisted electrochemical etching process for the formation of GaN porous structures. Pore linearity and depth controllability were strongly affected by the anode voltage. In addition, the use of light with an energy below the band gap played an important role in controlling the pore diameter. Spectro-electrochemical measurements revealed that the high electric field induced at the GaN/electrolyte interface caused a redshift of the photoabsorption edge. This specific phenomenon can be explained by a theoretical calculation based on the Franz-Keldysh effect. On the basis of the results of our experimental and theoretical analyze, we propose a formation model for GaN porous structures. We also note that the application of the Franz-Keldysh effect is useful in controlling the structural properties of GaN porous structures.
\end{abstract}

Keywords: porous structure, Gallium Nitride $(\mathrm{GaN})$, electrochemical etching, Franz-Keldysh effect

*Corresponding author. E-mail address: kumazaki@rciqe.hokudai.ac.jp

\section{Introduction}

Gallium nitride $(\mathrm{GaN})$ and its alloys are attractive building block materials for various functional devices, such as light-emitting diodes (LEDs), ${ }^{1,2)}$ laser diodes, ${ }^{3)}$ solar cells, ${ }^{4)}$ and so on. ${ }^{5,6}$ One promising approach for improving device performance is the formation of nanostructures with unique physical and optical features. Most reported GaN nanostructures have been formed using a selective-area growth ${ }^{7,8)}$ or dry etching process such as reactive-ion etching (RIE). ${ }^{9,10}$ ) There are, however, severe limitations on increasing the density of nanostructures, because most approaches use lithography to define the size and position of the nanostructures. Furthermore, when a dry etching process is involved, the etching damage induced by ion bombardment is considerable ${ }^{11-13)}$ and could significantly degrade the device performance.

One alternative approach is an electrochemical fabrication process, which can form various semiconductor nanostructures in a self-assembled fashion. The most well-known application of such an electrochemical process is the formation of porous structures by anodic etching, in which a high-density array of nanometer- or micrometer-sized pores is formed with high productivity over a large area on the semiconductor surface. ${ }^{14-18)}$ In addition, optical properties are significantly enhanced by the formation of porous structures. ${ }^{19,20)}$ These features are very promising for use in functional devices mentioned above.

The electrochemical conditions including applied bias and electrolyte solutions have been investigated with regard to the formation of $\mathrm{GaN}$ porous structures. $^{21)}$ Thus far, however, the controllability of structural properties such as the pore diameter and depth has been inadequate. ${ }^{22,23)}$ One reason for the difficulty in structural control is that a photoassisted electrochemical process is commonly used, and this formation process becomes more complicated as the illumination generates a supply of photocarriers. ${ }^{24)}$

In this study, therefore, we aimed to develop a photoassisted electrochemical etching process for forming $\mathrm{GaN}$ porous structures with better structural controllability. Spectro-electrochemical measurements were also conducted to clarify the relationship between photoabsorption and the electrochemical reaction.

\section{Methods}

An n-type GaN substrate $\left(N_{\mathrm{D}}=1 \times 10^{18} \mathrm{~cm}^{-3}\right)$ with a thickness of $400 \mu \mathrm{m}$ was used in this study. Gold $\mathrm{Au}$ ) was deposited at one corner of the back surface to supply an electrochemical current. A custom-made electrochemical cell was used to form porous structures and conduct spectro-electrochemical measurements, as shown schematically in Fig. 1. The electrochemical cell consisted of three electrodes: an n-type GaN working electrode (WE), a Pt counter electrode (CE), and a $\mathrm{Ag} / \mathrm{AgCl}$ reference electrode (RE). We used a mixture of $1 \mathrm{~mol} / \mathrm{L} \mathrm{H}_{2} \mathrm{SO}_{4}$ and $1 \mathrm{~mol} / \mathrm{L} \mathrm{H}_{3} \mathrm{PO}_{4}$ as an electrolyte. The $\mathrm{GaN}$ porous structures were formed under various

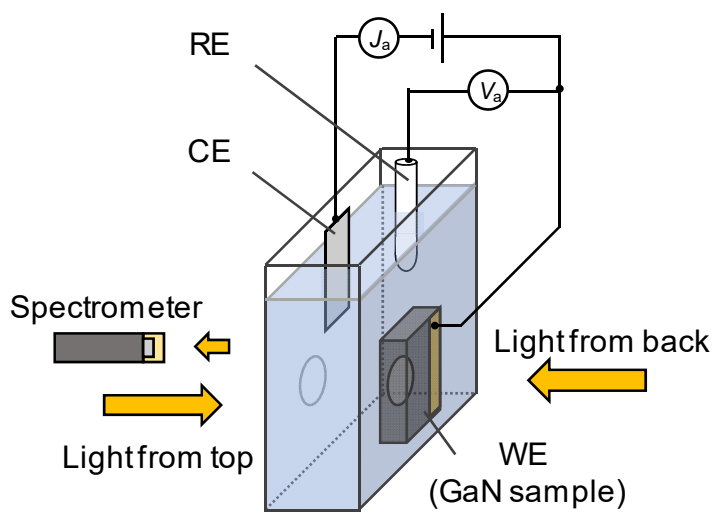

Fig. 1. Schematic illustration of the electrochemical setup used for both the formation of porous structures and the spectro-electrochemical measurements. 


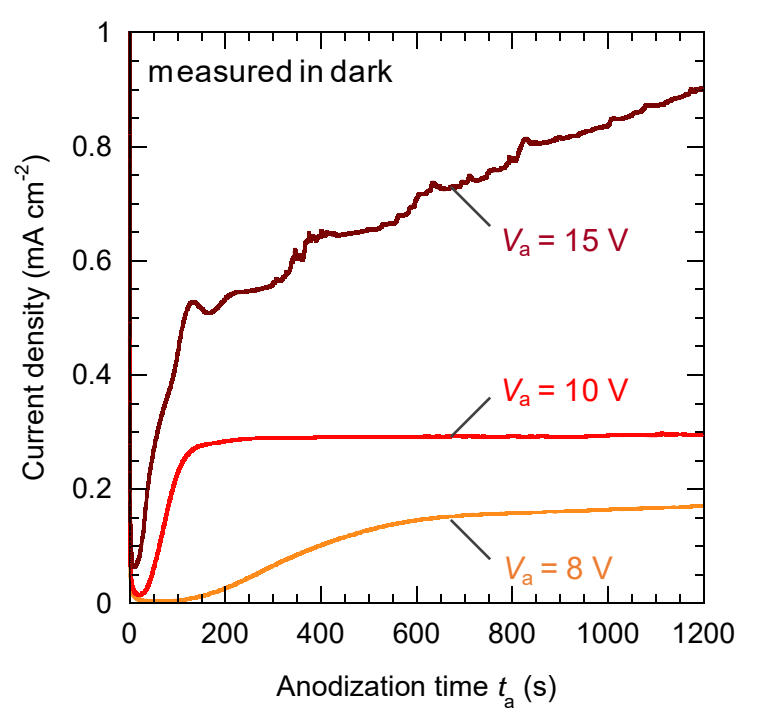

Fig. 2. Current-time curves obtained during anodization at various anode voltage $V_{\text {a }}$ values.

electrochemical conditions by varying such parameters as the anode voltage $V_{\mathrm{a}}$, anodization time $t_{\mathrm{a}}$, and light conditions. A xenon (Xe) lamp with an optical filter was used as the light source. The structural properties of the porous structures were evaluated by scanning electron microscopy (SEM) with a Hitachi SU-8010 system. The spectro-electrochemical properties were evaluated by measuring the transmittance of light irradiated from the back surface with an Ocean Optics HR4000CG-UV-NIR spectrometer, under a voltage applied in the same setup shown in Fig. 1.

\section{Results and discussion}

3.1 Formation of GaN porous structures

Before applying the photoassisted electrochemical process, the anode voltage condition was first optimized in the dark. Figure 2 shows current-time curves obtained during anodization at various anode voltage $V_{\text {a }}$ values. At the initial stage of electrochemical reactions, anodic currents increased for one hundred seconds or more. The slope of the curve increased with $V_{\mathrm{a}}$; in other words, the reaction rate increased with $V_{\mathrm{a}}$. In addition, we found that anodic currents saturated for the data obtained at $V_{\mathrm{a}}=8$ and $10 \mathrm{~V}$, but they kept increasing at $V_{\mathrm{a}}=15 \mathrm{~V}$. These results indicate that the reaction rates at $V_{\mathrm{a}}=8$ and $10 \mathrm{~V}$ become constant after one hundred seconds, whereas the reaction rate at $V_{\mathrm{a}}=15 \mathrm{~V}$ increases with anodization time.

Figures 3(a)-(c) show SEM images of GaN porous structures formed with $t_{\mathrm{a}}=1200 \mathrm{~s}$ and various anode voltages: (a) $V_{\mathrm{a}}=8 \mathrm{~V}$, (b) $V_{\mathrm{a}}=10 \mathrm{~V}$, and (c) $V_{\mathrm{a}}=$ $15 \mathrm{~V}$. The sample formed at $V_{\mathrm{a}}=8 \mathrm{~V}$ [Fig. 3(a)] had straight pores oriented perpendicular to the top surface, with pores branching from the straight pores. The sample formed at $V_{\mathrm{a}}=10 \mathrm{~V}$ [Fig. 3(b)] also had straight pores oriented perpendicular to the top surface, but the pore branching observed at $V_{\mathrm{a}}=8 \mathrm{~V}$ was significantly suppressed. The pore diameter and depth were estimated as approximately $16 \mathrm{~nm}$ and $1.3 \mu \mathrm{m}$, respectively, throughout the entire porous region. The general features observed here can be roughly explained in terms of two different, competing processes. The first process is pore formation in the preferential directions of weak bonds that are easily attacked by the electrolyte. The second
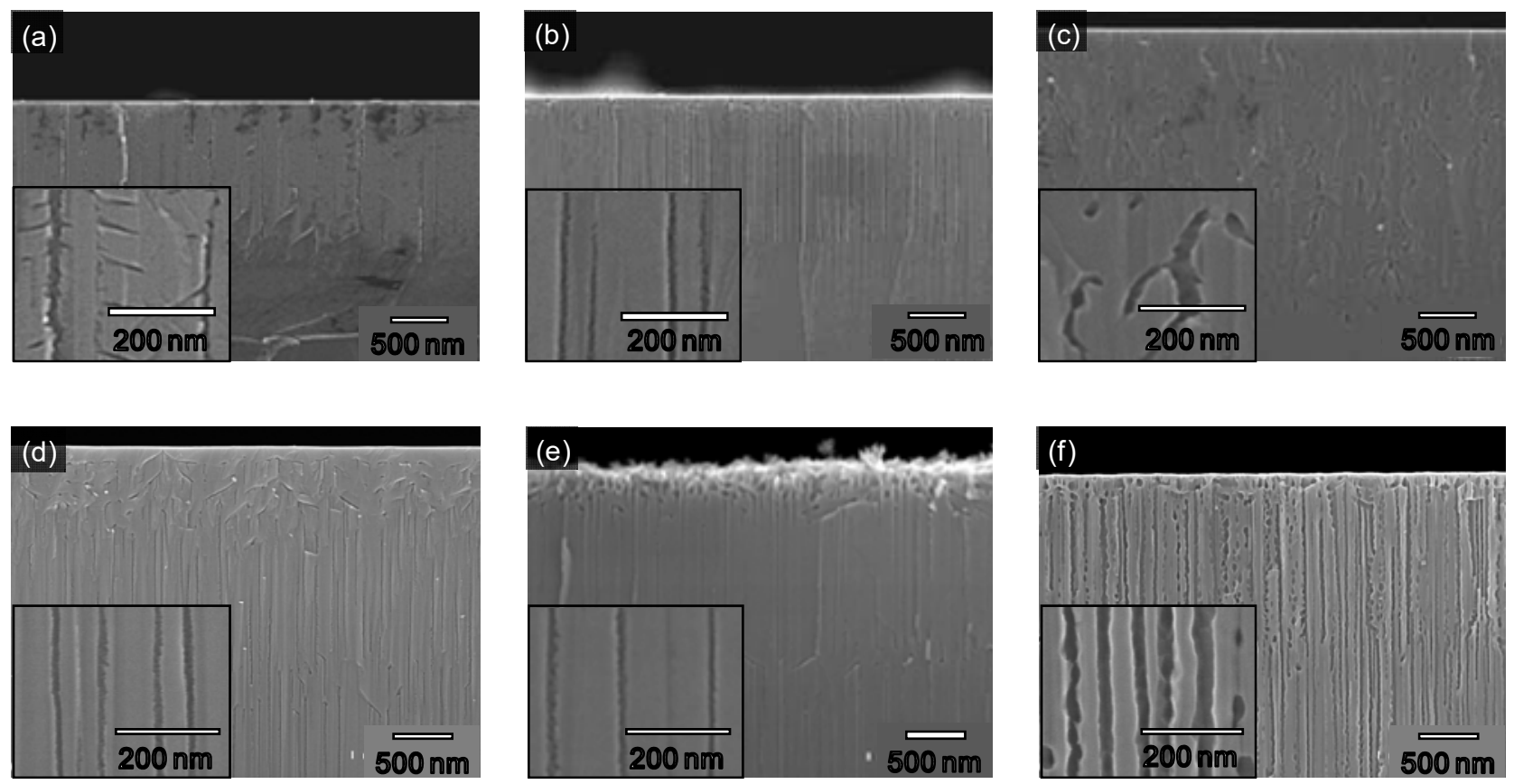

Fig. 3. Cross-sectional SEM images of GaN porous structures formed under various electrochemical conditions: (a) dark, $t_{\mathrm{a}}=1200 \mathrm{~s}$, $V_{\mathrm{a}}=8 \mathrm{~V}$; (b) dark, $t_{\mathrm{a}}=1200 \mathrm{~s}, V_{\mathrm{a}}=10 \mathrm{~V}$; (c) dark, $t_{\mathrm{a}}=1200 \mathrm{~s}, V_{\mathrm{a}}=15 \mathrm{~V} ;$ (d) dark, $t_{\mathrm{a}}=2400 \mathrm{~s}, V_{\mathrm{a}}=10 \mathrm{~V}$; (e) illuminated, $t_{\mathrm{a}}=2400 \mathrm{~s}$, $V_{\mathrm{a}}=10 \mathrm{~V}, h v=3.54 \mathrm{eV}$; (f) illuminated, $t_{\mathrm{a}}=2400 \mathrm{~s}, V_{\mathrm{a}}=10 \mathrm{~V}, h v=3.26 \mathrm{eV}$. 


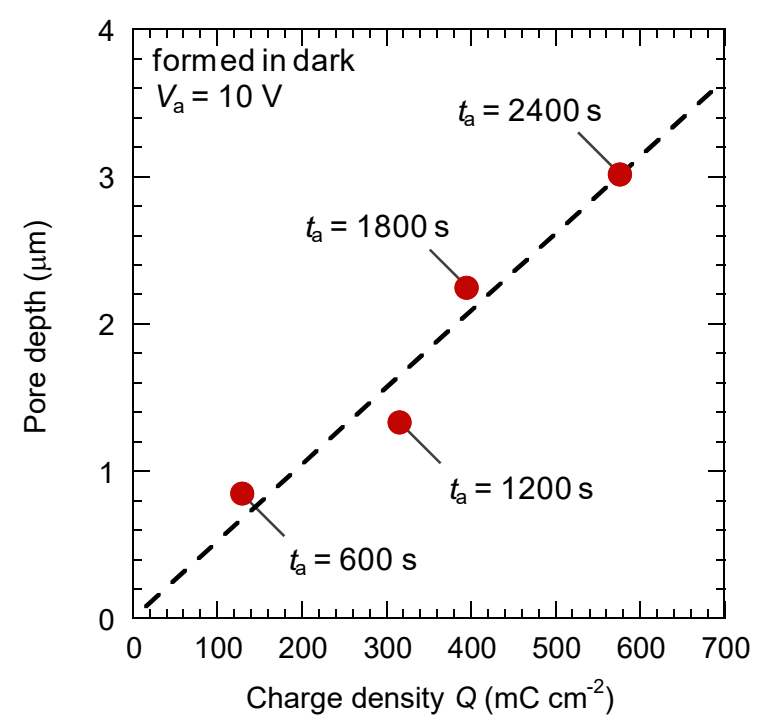

Fig. 4. Relationship between pore depth and density of charges, $Q$, passing through the working electrode during electrochemical etching.

process is the formation of straight pores along the direction of the electric field producing the holes required for the etching reaction. This tendency is enhanced at higher anodization voltages, as in the case of InP porous structures formed in the dark. ${ }^{25)}$ On the other hand, the pores formed at $V_{\mathrm{a}}=15 \mathrm{~V}$ [Fig. 3(c)] had diminished linearity and size uniformity. This was probably because the lateral etching of the pore walls was enhanced by the generation of holes in the walls. From these results, we can conclude that pore morphologies are markedly affected by $V_{\mathrm{a}}$, and that electrochemical etching at $V_{\mathrm{a}}=10$ $\mathrm{V}$ can achieve the formation of linear, uniform porous structures.

Figure 3(d) shows an SEM image of GaN porous structures formed with $t_{\mathrm{a}}=2400 \mathrm{~s}$ and $V_{\mathrm{a}}=10 \mathrm{~V}$. As compared with those of the sample shown in Fig. 3(b), the pore diameter and linearity of the structures shown in Fig. 3 remained unchanged, but the pore depth increased with $t_{\mathrm{a}}$. Figure 4 shows the relationship between pore depth and the density of charges, $Q$, passing through the working electrode during anodization. We found that the pore depth increased linearly with $Q$. Considering the proportional relationship between the amount of electrochemical reaction and $Q$, as described by Faraday's law, the electric charges contributed only to vertical etching, resulting in superior depth controllability. The pore diameter, however, could not be controlled throughout the electrochemical etching in the dark.

To investigate the feasibility of diameter control, the photoassisted electrochemical etching was next conducted in the front-side illumination mode. Figures 3(e) and 3(f) show SEM images of GaN porous structures formed at $V_{\mathrm{a}}=10 \mathrm{~V}$ and $t_{\mathrm{a}}=2400 \mathrm{~s}$, under monochromatic light with energy $h v$ values of (e) 3.54 and (f) $3.26 \mathrm{eV}$. Under illumination with $h v=3.54 \mathrm{eV}$, which is larger than the band gap $E_{\mathrm{g}}$ of $\mathrm{GaN}$, only pores near the top surface were affected by the light. In other words, the pore diameter increased only in the top region,

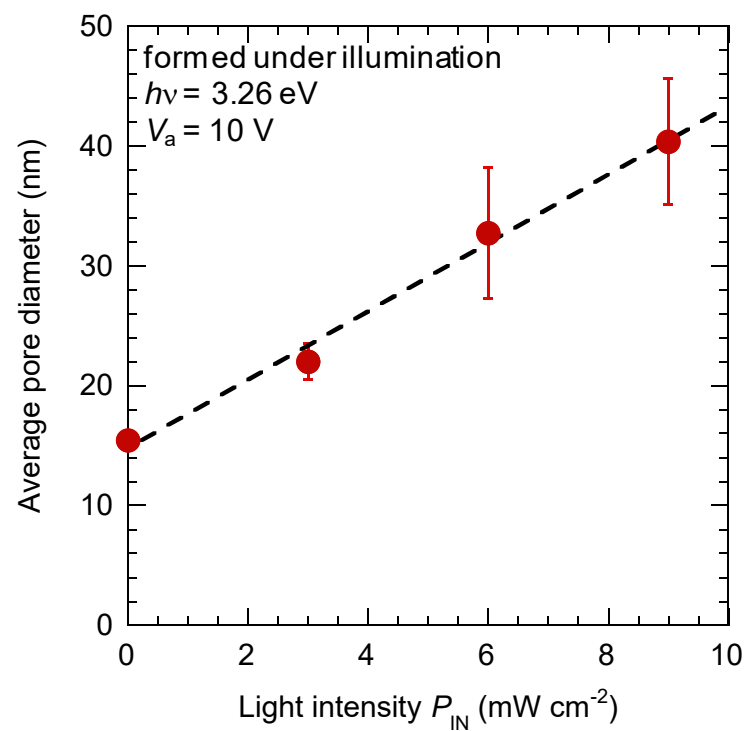

Fig. 5. Relationship between pore diameter and light intensity $P_{\mathrm{IN}}$ with $h v=3.26 \mathrm{eV}$.

to a depth of about $200 \mathrm{~nm}$, as compared with that of the samples formed in the dark. From SEM observations, an average pore diameter of $50 \mathrm{~nm}$ was obtained in the top region, which is approximately $30 \mathrm{~nm}$ larger than the pore diameter in the deeper region. On the other hand, under illumination with $h v=3.26 \mathrm{eV}\left(<E_{\mathrm{g}}\right)$, the pore diameter increased to approximately $40 \mathrm{~nm}$ throughout the entire porous layer. Figure 5 shows the average pore diameter plotted as a function of the light intensity $P_{\mathrm{IN}}$. We found that the pore diameter increased linearly with $P_{\mathrm{IN}}$. These results clearly indicate that using light with $h v$ below the band gap of $\mathrm{GaN}$ enables the tuning of the diameter without diminishing the linearity or depth controllability of the pores.

\subsection{Spectro-electrochemical properties}

To clarify how the photoabsorption process affects electrochemical reactions, spectro-electrochemical measurements were conducted on planar GaN substrates. Figure 6(a) shows transmittance spectra for $T_{0 \mathrm{~V}}$ and $T_{10 \mathrm{~V}}$ obtained by applying anode voltages of $V_{\mathrm{a}}=0$ and $10 \mathrm{~V}$, respectively, in the electrochemical setup shown in Fig. 1. We could observe a strong dependence of the transmittance on $V_{\text {a }}$ at around $3.3 \mathrm{eV}$. The transmittance difference $T_{0 \mathrm{~V}}-T_{10 \mathrm{~V}}$ is plotted in Fig. 6(b) as a function of the photon energy $h v$. The difference was almost zero at energies higher than $E_{\mathrm{g}}$ because of absorption by the bulk GaN. On the other hand, larger differences could be observed at energies below $E_{\mathrm{g}}$, reaching a maximum at around $3.3 \mathrm{eV}$ and decreasing as $h v$ decreased. These results indicate that photoabsorption near the band edge can be enhanced by the electric field mostly applied at the GaN/electrolyte interface.

One possible phenomenon to explain the above results is the Franz-Keldysh effect, which causes the redshift of the absorption edge under a high electric field, leading to the occurrence of absorption below the bulk band gap $E_{\mathrm{g}}{ }^{26,27)}$ Generally, a high electric field is applied 
(a)

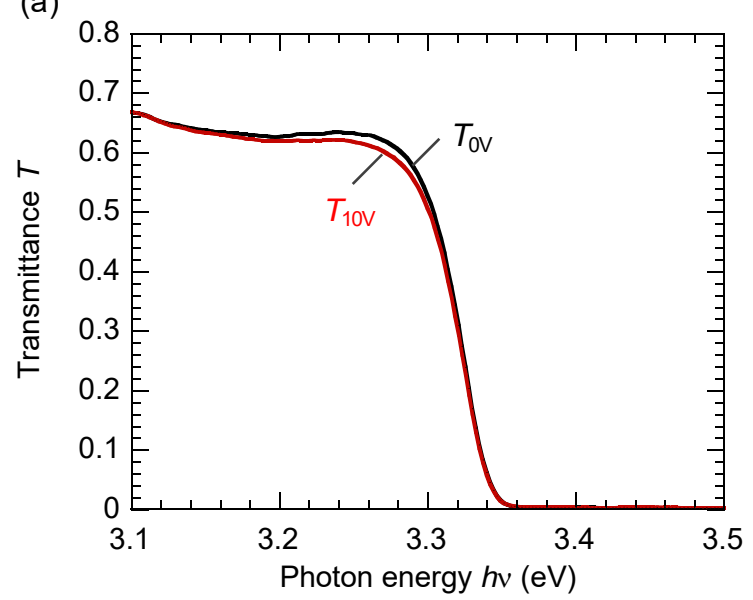

(b)

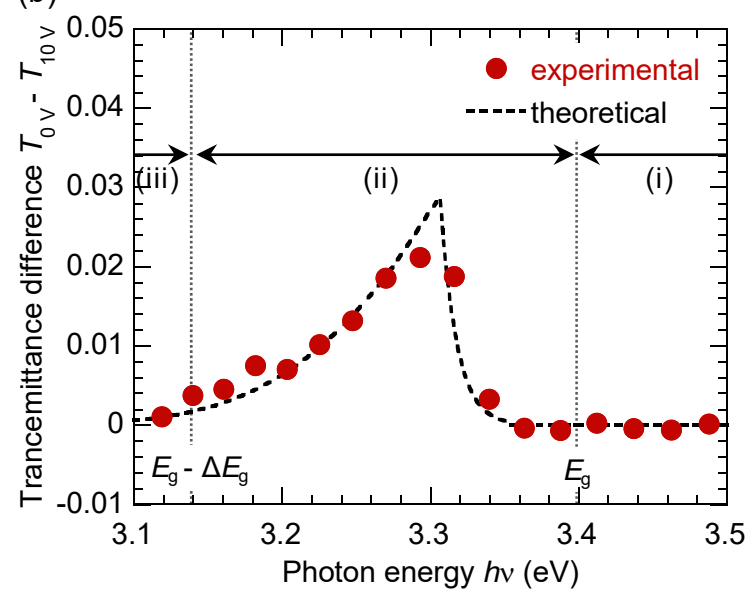

Fig. 6. (a) Transmittance spectra of a GaN planar electrode, obtained by applying anode voltages of $V_{\mathrm{a}}=0$ and $10 \mathrm{~V}$ in the electrochemical setup shown in Fig. 1. (b) Transmittance difference $T_{0 \mathrm{~V}}-T_{10 \mathrm{v}}$ plotted as a function of the photon energy $h \mathrm{v}$.

by the formation of a potential barrier of about $1.0 \mathrm{eV}$ at the GaN/electrolyte interface ${ }^{28,29)}$ When the doping density is as high as $10^{18} \mathrm{~cm}^{-3}$, for example, the internal electric field in a thin depletion layer with a width of several dozen nanometers reaches $5 \times 10^{5} \mathrm{~V} / \mathrm{cm}$. In such a situation, the electron and hole wave functions become Airy functions, which extend into the band gap, enabling band-to-band transition at energies below $E_{\mathrm{g}}$. In the depletion region, the Franz-Keldysh relative absorption coefficient $\alpha$ can be approximated as $^{30,31)}$

$$
\alpha \sim \alpha_{0} \exp \left(-\left|\frac{h v-E_{\mathrm{g}}}{\Delta E_{\mathrm{g}}}\right|^{\frac{3}{2}}\right) .
$$

Here, $\alpha_{0}$ is the absorption coefficient at $h v=E_{\mathrm{g}}$, and $\Delta E_{\mathrm{g}}$ is the redshift energy of the absorption edge, expressed by

$$
\Delta E_{\mathrm{g}}=\frac{2}{3} \frac{(e \hbar \xi)^{\frac{3}{2}}}{(m *)^{\frac{1}{3}}},
$$

where $q, \hbar, \xi$, and $m^{*}$ are the elementary charge, Dirac's constant, electric field, and effective mass, respectively. The transmittance $T$ is expressed as a function of the reflectance $R$, absorption coefficient $\alpha$, and optical path length $x$, as follows:

$$
T=(1-R) \exp (-\alpha x) .
$$

Through calculations using Eqs. (1)-(3), we estimated the transmittance difference $T_{0 \mathrm{~V}}-T_{10 \mathrm{~V}}$, plotted in Fig. 6(b) with a dotted line. The experimental data are very consistent with the prediction of the Franz-Keldysh effect. In the case of illumination with $h v>E_{\mathrm{g}}$ [case (i)], $T_{0 \mathrm{~V}}-T_{10 \mathrm{~V}}$ was almost zero since all photons were absorbed near the surface. For illumination with $E_{\mathrm{g}}-\Delta E_{\mathrm{g}}$ $<h v<E_{\mathrm{g}}$ [case (ii)], the photons penetrated through the bulk $\mathrm{GaN}$ but were absorbed at the GaN/electrolyte interface only under a high electric field because of the Franz-Keldysh effect. In that situation, $T_{0 \mathrm{~V}}-T_{10 \mathrm{~V}}$ increased up to an observable level, as shown in Fig. 6(b). Finally, for illumination with $h v<E_{\mathrm{g}}-\Delta E_{\mathrm{g}}$ [case (iii)], since photons were not absorbed either in the bulk GaN or at the GaN/electrolyte interface, $T_{0 \mathrm{~V}}-T_{10 \mathrm{~V}}$ decreased again. These results suggest that light with $E_{\mathrm{g}}-\Delta E_{\mathrm{g}}<h v$ $<E_{\mathrm{g}}$ plays an important role in the photoassisted electrochemical process.

\subsection{Formation mechanism of GaN porous structures}

To investigate the possibility of applying the Franz-Keldysh effect, we calculated the potential distribution of $\mathrm{GaN}$ porous structures in an electrolyte by solving a 3D Poisson equation. Figure 7(a) shows the potential distribution of the conduction band minimum measured from the Fermi level, $E_{\mathrm{C}}-E_{\mathrm{F}}$, with the solid lines indicating contours at $0.2,0.4,0.6,0.8$, and $1.0 \mathrm{eV}$. The pore pitch, depth, and potential difference between the $\mathrm{GaN}$ and electrolyte were specified as $25 \mathrm{~nm}, 50 \mathrm{~nm}$, and $1.0 \mathrm{eV}$, respectively. Although no external voltage is applied, the potential distribution is strongly modified near the pore tips, where the contour intervals become narrow. Figure 7(b) shows cross-sectional potential distributions obtained at the top surface (line i) and pore tips (line ii). The potential variation at the pore tips is much larger than that at the top surface, reflecting the high electric field applied to the tips.

On the basis of the above discussion, we propose a formation model for GaN porous structures. For formation in the dark, the electrochemical etching is performed by the carriers generated by the avalanche effect induced by the concentrated electric field at the pore tips. Since the electric fields at the pore walls and top surface are small, the electrochemical etching proceeds only in the depth direction at the pore tips. That is why the process of forming $\mathrm{GaN}$ porous structures in the dark can achieve superior linearity and depth controllability but cannot vary the pore diameter. Under illumination with $h v>E_{\mathrm{g}}$, photons are preferentially absorbed near the top surface because of band-edge absorption. Photogenerated carriers are then transferred to the top surface and pore walls by the electric field, and 

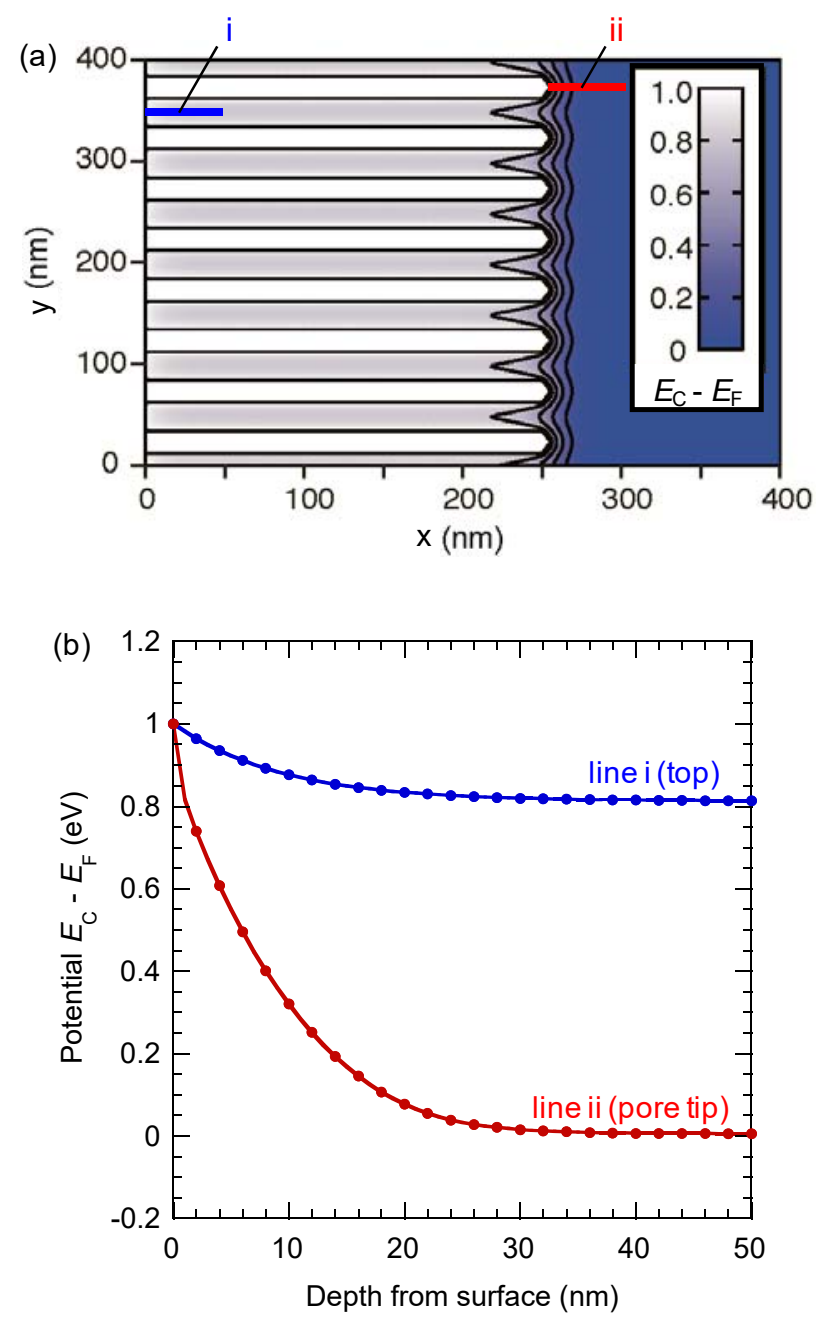

Fig. 7. (a) Potential distribution in $\mathrm{GaN}$ porous structures drawn with contour levels at $0.2 .0 .4,0.6,0.8$, and $1.0 \mathrm{eV}$, and (b) the cross-sectional potential distribution at the top surface (line i) and pore tips (line ii) obtained by solving 3D Poisson equation.

contribute to the electrochemical etching. This explains the larger pore region with a thickness of $200 \mathrm{~nm}$, a value consistent with the light penetration depth in GaN [see Fig. 3(e)]. Under illumination with $E_{\mathrm{g}}-\Delta E_{\mathrm{g}}<h v<E_{\mathrm{g}}$, however, photons are transmitted in the top region but absorbed only at the pore tips where a high electric field is applied. In this situation, the photocarriers generated by the Franz-Keldysh effect are consumed at the pore tips and assist the electrochemical etching. As seen from Fig. 5 , in fact, the pore diameter increased with the light intensity, as the number of carriers contributing to the electrochemical etching increased. This formation model can consistently explain both the experimental results and the theoretical model, enabling us to conclude that the application of the Franz-Keldysh effect is useful in controlling the structural properties of $\mathrm{GaN}$ porous structures.

\section{Conclusions}

Porous structures in $\mathrm{GaN}$ were formed by electrochemical etching under various conditions. Pore linearity and depth controllability were improved by selecting an appropriate anode voltage $V_{\mathrm{a}}$. In addition, we succeeded in controlling the pore diameter by using light with $h v$ below the band gap. Spectro-electrochemical measurements revealed that the high electric field induced at the GaN/electrolyte interface caused a redshift of the photoabsorption edge. This specific phenomenon could be consistently explained by a theoretical calculation based on the Franz-Keldysh effect. On the basis of the results of our experimental and theoretical analyze, we proposed a formation model for $\mathrm{GaN}$ porous structures. We also concluded that the application of the Franz-Keldysh effect is useful in controlling the structural properties of GaN porous structures.

\section{Acknowledgement}

This work was supported in part by a Grant-in-Aid for JSPS Fellows 14J01371, a Grant-in-Aid for Scientific Research (B) 25289079, and a Grant-in-Aid for Challenging Exploratory Research 15K13937, from the Japan Society for the Promotion of Science (JSPS).

\section{References}

1) S. Nakamura, T. Mukai, and M. Senoh, Jpn. J. Appl. Phys. 30, L1998 (1991).

2) I. Akasaki and H. Amano, Jpn. J. Appl. Phys. 45, 9001 (2006)

3) S. Nakamura, M. Senoh, S. Nagahara, N. Iwasa, T. Yamada, T. Matsushita, H. Kiyoku, and Y. Sugimoto, Jpn. J. Appl. Phys. 35, L74 (1996)

4) C. J. Neufeld, N. G. Toledo, S. C. Cruz, M. Iza, S. P. DenBaars, and U. K. Mishra, Appl. Phys. Lett. 93, 143502 (2008).

5) K. Fujii, T. Karasawa, and K. Ohkawa, Jpn. J. Appl. Phys. 44, L543 (2005).

6) S. Yotsuhashi, M. Deguchi, Y. Zenitani, R. Hinogami, H. Hashiba, Y. Yamada, and K. Ohkawa, Appl. Phys. Express 4, 117101 (2011).

7) Y. D. Wang, K. Y. Zang, and S. J. Chua, J. Appl. Phys. 100, 054306 (2006).

8) I. Waki, D. Cohen, R. Lal, U. Mishra, S. P. DenBaars, and S. Nakamura, Appl. Phys. Lett. 91, 093519 (2007).

9) H. Ono, Y. Ono, K. Kasahara, J. Mizuno, and S. Shoji, Jpn. J. Appl. Phys. 47, 933 (2008).

10) W. M. Zhou, G. Q. Min, Z. T. Song, J. Zhang, Y. B. Liu, and J. P. Zhang, Nanotechnology 21, 205304 (2010). 11) E. D. Haberer, C. H. Chen, A. Abare, M. Hansen, S. Denbaars, L. Coldren, U. Mishra, and E. L. Hu, Appl. Phys. Lett. 76, 3941 (2000).

12) R. Dimitrov, V. Tilak, W. Yeo, B. Green, H. Kim, J. Smart, E. Chumbes, J. R. Shealy, W. Schaff, L. F. Eastman, C. Miskys, O. Ambacher, and M. Stutzmann, Solid-State Electron. 44, 1361 (2000).

13) F. A. Khan, L. Zhou, V. Kumar, and I. Adesida, J. Vac. Sci. Technol. B 19, 2926 (2001).

14) A. Uhlir, Bell Syst. Tech. J. 35, 333 (1956).

15) V. Lehmann, J. Electrochem. Soc. 140, 2836 (1993).

16) B. H. Erné, D. Vanmaekelbergh, and J. J. Kelly, J. Electrochem. Soc. 143, 305 (1996).

17) H. Hasegawa and T. Sato, Electrochim. Acta 50, 3015 
(2005).

18) H. Asoh, K. Nishio, M. Nakao, T. Tamamura, and H. Masuda, J. Electrochem. Soc. 148, B152 (2001).

19) T. Sato, N. Yoshizawa, and T. Hashizume, Thin Solid Films 518, 4399 (2010).

20) Y. Kumazaki, T. Kudo, Z. Yatabe, and T. Sato, Appl. Surf. Sci. 279, 116 (2013).

21) S. F. Cheah, S. C. Lee, S. S. Ng, F. K. Yam, H. Abu Hassan, and Z. Hassan, J. Lumin. 159, 303 (2015).

22) W. J. Tseng, D. H. van Dorp, R. R. Lieten, P. M. Vereecken, and G. Borghs, J. Phys. Chem. C 118, 29492 (2014).

23) Y. Kumazaki, A. Watanabe, Z. Yatabe, and T. Sato, J. Electrochem. Soc. 161, H705 (2014).

24) A. Watanabe, Y. Kumazaki, Z. Yatabe, and T. Sato, ECS Electrochem. Lett. 4, H11 (2015).

25) T. Sato, T. Fujino, and H. Hasegawa, Appl. Surf. Sci. 252, 5457 (2006).

26) W. Franz, Z. Naturforsch. A 13, 484 (1958).

27) L. V. Keldysh, Sov. Phys. JETP 6, 763 (1958).

28) S. S. Kocha, M. W. Peterson, D. J. Arent, J. M. Redwing, M. A. Tischler, and J. A. Turner, J. Electrochem. Soc. 142, L238 (1995).

29) I. M. Huygens, K. Strubbe, and W. P. Gomes, J. Electrochem. Soc. 147, 1797 (2000).

30) H. Y. Peng, M. D. McCluskey, Y. M. Gupta, M. Kneissl, and N. M. Johnson, Appl. Phys. Lett. 82, 2085 (2003).

31) A. Cavallini, L. Polenta, and M. Rossi, Nano Lett. 7, 2166 (2007). 